\title{
Fuzzy Spatial Relations between Vague Regions
}

\author{
Steven Schockaert, Chris Cornelis, Martine De Cock, Etienne E. Kerre
}

\begin{abstract}
Various intelligent systems rely heavily on formalisms for spatial representation and reasoning. However, it is widely recognized that real-world regions are seldom characterized by a precisely defined boundary. This paper proposes a generalization of the Region Connection Calculus (RCC) which allows to define spatial relations between vague regions. To this end, spatial relations are modelled as fuzzy relations. To support spatial reasoning based on these relations, we give some important properties and a transitivity table. Furthermore, we show how imprecise spatial information can be modelled in our approach when vague regions are represented as fuzzy sets.
\end{abstract}

Index Terms - Spatial Reasoning, Region Connection Calculus, Fuzzy Relation, Approximate Equality.

\section{INTRODUCTION}

Spatial relations play a fundamental role in various application areas, ranging from GIS systems to robotics and image understanding. Although spatial relations are often modelled in a quantitative way, in some cases a more qualitative approach is desirable. This may be because a fully quantitative (numeric) representation is computationally too expensive, or because our knowledge of a spatial scene is incomplete, (e.g. based on a description in natural language). Both the Region Connection Calculus (RCC) [1] and the 9-intersection model [2] provide a formal characterization of qualitative spatial relations; we will focus on the former.

The definitions of the spatial relations in the RCC rely on a primitive two-valued relation $C$ which is assumed (axiomatized) to be reflexive and symmetric. Intuitively, $C(a, b)$ means that regions $a$ and $b$ are connected. The other relations of the RCC are defined as in Table I, where the quantifications range over the universe $U$ of regions.

It is often assumed that regions are regularly closed sets of points. That is, for $\mathcal{P}$ a universe of points equiped with a topology, a region $a$ in $\mathcal{P}$ is a subset of $\mathcal{P}$ for which $c l(i(a))=a$, where $c l$ and $i$ denote the topological closure and interior respectively. Then the intended interpretation of $C$ is that for two regions $a$ and $b, C(a, b)$ holds iff $a$ and $b$ share a common point. The intended interpretation of $D R$ (discrete from), $P O$ (partially overlaps with), $E C$ (externally connects with), TPP (tangential proper part of) and NTPP (non-tangential proper part of) is illustrated in Figure 1. For two regions $a$ and $b$, the intended interpretation of $O$ (overlaps with) is that $O(a, b)$ holds iff the interiors of $a$ and $b$ share a common point.

Various properties between the spatial relations of the

Steven Schockaert*, Chris Cornelis ${ }^{\dagger}$, Martine De Cock and Etienne E. Kerre are with the Department of Applied Mathematics and Computer Science, Ghent University, Krijgslaan 281 (S9), B-9000 Gent, Belgium (e-mail: \{Steven.Schockaert, Chris.Cornelis, Martine.DeCock, Etienne.Kerre\}@UGent.be.)

* Research assistant of the Fund for Scientific Research - Flanders

${ }^{\dagger}$ Post-doctoral fellow of the Fund for Scientific Research - Flanders

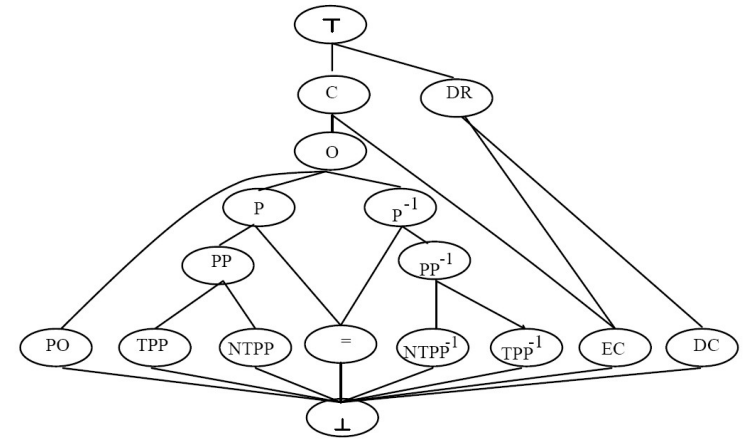

Fig. 2. Subsumption hierarchy of the RCC relations [1]

RCC are summarized in Figure 2: a relation holds iff at least one of the relations mentioned in its child nodes holds [1]. For instance, if $\operatorname{PP}(a, b)$ then $\operatorname{TPP}(a, b)$ or $N T P P(a, b)$, if $P(a, b)$ then $P P(a, b)$ or $E Q(a, b)$; if $P P(a, b)$ then $P(a, b)$, if $O(a, b)$ then $C(a, b)$, etc. It can also be shown that the relations $D C, E Q, E C, P O, T P P$, $N T P P, T P P^{-1}$ and $N T P P^{-1}$ are jointly exclusive and pairwise disjoint (JEPD). The RCC restricted to these eight relations is called the RCC-8. Similarly, the relations $D R, E Q, P O, P P, P P^{-1}$ are a set of JEPD relations, which constitute the RCC-5.

One major limitation of the RCC as such is that its spatial relations are crisp: either they hold or they do not hold. In this paper we extend the RCC by providing generalized definitions of the spatial relations as fuzzy relations. This allows to express the degree to which a particular spatial relation between two regions holds. In accordance with the RCC, our spatial relations are defined w.r.t. an arbitrary reflexive and symmetric fuzzy relation $C$. As a consequence, the new definitions can still be used regardless of whether the regions are vague or not, and regardless of how the regions are represented. Yet, in the particular case where (vague) regions are represented by (fuzzy) sets of points, and $C$ is based on "nearness" between the points of the regions, we are able to represent imprecise spatial relations between regions such as " $a$ is located in the heart of $b$ ", which cannot be modelled in the RCC, not even when $a$ and $b$ are crisp regions.

The paper is structured as follows: in Section II, we recall some basic notions from fuzzy set theory and fuzzy relational calculus, while Section III reviews related work on spatial relations between vague regions. Then, in Section IV we introduce the definitions of our fuzzy spatial relations. We also show that generalized versions of the RCC properties can be maintained for our definitions, and that they support spatial reasoning. Next, in Section V, we focus on the specific case where crisp regions are represented as sets of points, vague regions as fuzzy sets of 
TABLE I

Definition of the Spatial Relations of the RCC. $a$ And $b$ ARe Regions. Quantifications Range over the Universe $U$ of Regions.

\begin{tabular}{lll}
\hline Name & Definition & \\
\hline disconnected from & $D C(a, b)$ & $\equiv \neg C(a, b)$ \\
part of & $P(a, b)$ & $\equiv(\forall c \in U)(C(c, a) \Rightarrow C(c, b))$ \\
proper part of & $P P(a, b)$ & $\equiv P(a, b) \wedge \neg P(b, a)$ \\
coincides with & $E Q(a, b)$ & $\equiv P(a, b) \wedge P(b, a)$ \\
overlaps with & $O(a, b)$ & $\equiv(\exists c \in U)(P(c, a) \wedge P(c, b))$ \\
discrete from & $D R(a, b)$ & $\equiv \neg O(a, b)$ \\
partially overlaps with & $P O(a, b)$ & $\equiv O(a, b) \wedge \neg P(a, b) \wedge \neg P(b, a)$ \\
externally connects with & $E C(a, b) \quad \equiv C(a, b) \wedge \neg O(a, b)$ \\
tangential proper part of & $T P P(a, b)$ & $\equiv P P(a, b) \wedge(\exists c \in U)(E C(c, a) \wedge E C(c, b))$ \\
non-tangential proper part of & $N T P P(a, b) \equiv P P(a, b) \wedge \neg(\exists c \in U)(E C(c, a) \wedge E C(c, b))$ \\
\hline
\end{tabular}

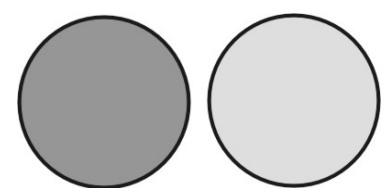

(a) $D R$

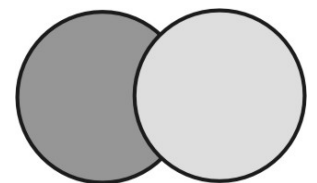

(b) $P O$

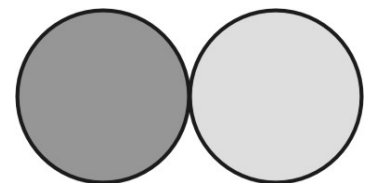

(c) $E C$

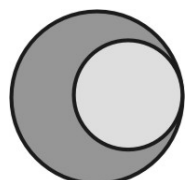

(d) $T P P$

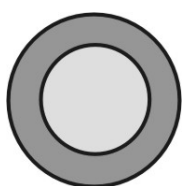

(e) NTPP

Fig. 1. Intended meaning of some of the spatial relations

points, and the fuzzy relation $C$ is based on a particular measure of approximate equality. Finally, in Section VI we round up the paper by outlining future work.

\section{PRELIMinaries}

A fuzzy set [3] $A$ in a universe $X$ is defined as a mapping from $X$ to the unit interval $[0,1]$. For $x$ in $X, A(x)$ is called the membership degree of $x$ in $A$. If there exists $x$ in $X$ such that $A(x)=1, A$ is called normalised.

A fuzzy set $R$ in $X \times X$ is called a fuzzy relation in $X$. $R$ is called reflexive iff $R(x, x)=1$ for all $x$ in $X$, and irreflexive iff $R(x, x)=0$ for all $x$ in $X$. It is symmetric iff $R(x, y)=R(y, x)$ for all $x$ and $y$ in $X$. The inverse of a fuzzy relation $R$ in $X$ is the fuzzy relation $R^{-1}$ in $X$ defined for all $x$ and $y$ in $X$ by $R^{-1}(y, x)=R(x, y)$.

Classical set theory is linked to binary logic in the sense that set operators such as intersection and union are usually defined in terms of logical connectives. In the same way, fuzzy set theory is linked with a generalization of binary logic in which the logical operators are defined on the unit interval. To generalize the logical conjunction to the unit interval $[0,1]$, we have a large class of $[0,1]^{2}-[0,1]$ mappings called t-norms at our disposal. A t-norm $T$ is defined as a symmetric, associative, increasing $[0,1]^{2}-[0,1]$ mapping satisfying the boundary condition $T(x, 1)=x$ for all $x$ in $[0,1]$. Some common t-norms are the minimum $T_{M}$, the product $T_{P}$ and the Lukasiewicz t-norm $T_{W}$, defined by

$$
\begin{aligned}
T_{M}(x, y) & =\min (x, y) \\
T_{P}(x, y) & =x \cdot y \\
T_{W}(x, y) & =\max (0, x+y-1)
\end{aligned}
$$

for all $x$ and $y$ in $[0,1]$. In the same way, logical disjunction can be generalized by t-conorms, i.e. symmetric, associative, increasing $[0,1]^{2}-[0,1]$ mappings $S$ satisfying $S(0, x)=x$ for all $x$ in $[0,1]$. Common t-conorms are the maximum $S_{M}$, the probabilistic sum $S_{P}$, and the Łukasiewicz t-conorm $S_{W}$, defined by

$$
\begin{array}{cc}
S_{M}(x, y) & =\max (x, y) \\
S_{P}(x, y) & =x+y-x y \\
S_{W}(x, y) & =\min (1, x+y)
\end{array}
$$

for all $x$ and $y$ in $[0,1]$. Next, the negation of an element $x$ in $[0,1]$ is commonly defined by $1-x$. Finally, a $[0,1]^{2}-[0,1]$ mapping $I$ which is decreasing in the first and increasing in the second argument and which satisfies $I(0,0)=I(0,1)=I(1,1)=1$ and $I(1,0)=0$ is called an implicator; implicators generalize the logical implication to the unit interval. There exist several ways to obtain implicators. For instance, let $S$ be an arbitrary t-conorm; it can be shown that the mapping $I_{S}$, defined for $x$ and $y$ in $[0,1]$ by

$$
I_{S}(x, y)=S(1-x, y)
$$

is an implicator, which is called the strong implicator of $S$. For example, the strong implicator corresponding to $S_{M}$ is defined by

$$
I_{S_{M}}(x, y)=\max (1-x, y)
$$


for all $x$ and $y$ in $[0,1]$. On the other hand, let $T$ be an arbitrary t-norm; it can be shown that the mapping $I_{T}$, defined for $x$ and $y$ in $[0,1]$ by

$$
I_{T}(x, y)=\sup \{\lambda \mid \lambda \in[0,1] \text { and } T(x, \lambda) \leq y\}
$$

is an implicator, which is called the residual implicator of $T$. For example, the residual implicator corresponding to the Eukasiewicz t-norm $T_{W}$ is defined by

$$
I_{T_{W}}(x, y)=\min (1,1-x+y)
$$

for all $x$ and $y$ in $[0,1]$. For convenience, we will write $I_{W}$ instead of $I_{T_{W}}$ in the remainder of this paper.

Next we recall some important constructs from fuzzy relational calculus. The direct image $A \uparrow_{T} R$ (w.r.t. a t-norm $T$ ) and the superdirect image $A \downarrow_{I} R$ (w.r.t. an implicator $I$ ) of a fuzzy set $A$ in $X$ under a fuzzy relation $R$ in $X$ are the fuzzy sets $R \uparrow_{T} A$ and $R \downarrow_{T} A$ in $X$ defined by

$$
\begin{aligned}
& \left(R \uparrow_{T} A\right)(y)=\sup _{x \in X} T(R(x, y), A(x)) \\
& \left(R \downarrow_{I} A\right)(y)=\inf _{x \in X} I(R(x, y), A(x))
\end{aligned}
$$

for all $y$ in $X$. When there is no cause for confusion, we will usually omit the subscripts in (11) and (12). For notational convenience, we introduce the following abbreviations:

$$
\begin{aligned}
R \uparrow_{T} \uparrow_{T} A & =R \uparrow_{T}\left(R \uparrow_{T} A\right) \\
R \downarrow_{I} \downarrow_{I} A & =R \downarrow_{I}\left(R \downarrow_{I} A\right) \\
R \uparrow_{T} \downarrow_{I} A & =R \uparrow_{T}\left(R \downarrow_{I} A\right) \\
R \downarrow_{I} \uparrow_{T} A & =R \downarrow_{I}\left(R \uparrow_{T} A\right)
\end{aligned}
$$

A fuzzy set $A$ is called $R$-closed iff $R \downarrow_{I} \uparrow_{T} A=A$.

The degree of overlap overl $(A, B)$ between two fuzzy sets $A$ and $B$ in $X$ is defined as

$$
\operatorname{overl}(A, B)=\sup _{x \in X} T(A(x), B(x))
$$

where $T$ is a t-norm; (17) expresses the degree to which there exists an element of $X$ that is both contained in $A$ and in $B$. In the same way, the degree of inclusion $\operatorname{incl}(A, B)$ of $A$ in $B$ is defined as

$$
\operatorname{incl}(A, B)=\inf _{x \in X} I(A(x), B(x))
$$

where $I$ is an implicator; (18) expresses the degree to which all elements of $X$ that are contained in $A$, are also contained in $B$. The degree of overlap and the degree of inclusion both express some (fuzzy) relationship between two fuzzy sets. Relatedness measures [4] are a more general notion which have (17) and (18) as special cases. In this paper we will use

$$
A \circ_{T} R \circ_{T} B=\sup _{x \in X} T\left(A(x), \sup _{y \in X} T(B(x), R(x, y))\right)
$$

where $A$ and $B$ are fuzzy sets in $X, R$ is a fuzzy relation in $X$ and $T$ is a t-norm; (19) expresses the degree to which there is an element of $A$ that is in relationship (w.r.t. $R$ ) with an element of $B$.

\section{RELATED WORK}

It has been widely recognized that in the real world, geographic regions tend to be vague (e.g. [5],[6],[7],[8]). How vague geographic regions should be modelled, however, has not been entirely uncontroversial.

Most definitions of spatial relations between vague regions extend either the RCC or the 9-intersection model by treating a vague region $a$ as being composed of two crisp regions: one region $\underline{a}$ which consists of the points that are definitely in the vague region, and one region $\bar{a}$ whose complement consists of the points that are definitely not in the vague region. The region defined by $\bar{a} \backslash \underline{a}$ (provided $\underline{a}$ is a proper part of $\bar{a}$ ) consists of the points for which it is hard to tell whether they are in the vague region or not.

A well-known example is the egg-yolk calculus [9] which is based on the RCC. In [10], a similar approach, based on the notion of a thick boundary, is proposed which is an extension of the 9-intersection model. Both models cause a significant increase in the number of possible relations: 46 and 44 relations respectively. For example, instead of specifying that two regions $a$ and $b$ overlap, we may specify that $\bar{a}$ and $\underline{b}$ overlap (but not $\underline{a}$ and $\underline{b}$ ), or that $\bar{a}$ and $\bar{b}$ overlap, or that $\underline{a}$ and $\underline{b}$ overlap, etc. where $\underline{a}$ and $\bar{a}$ (respectively $\underline{b}$ and $\bar{b}$ ) represent the yolk and the egg of $a$ (respectively $b$ ).

Another possibility, which is adopted in [11], is to stay with the 8 or 5 spatial relations of the RCC- 8 or RCC5 respectively, but to use three-valued relations instead of classical two-valued relations. Taking this idea one step further, we can generalize the RCC relations to many-valued, and fuzzy, relations.

In [5] and [8], the use of supervaluation semantics for the representation of vague regions is advocated. As pointed out in [5], it is useful to distinguish between conceptual vagueness and Sorites vagueness. Conceptual vagueness is closely related to ambiguity. A prototypical example is the definition of a forest [5]: should a forest be self-connected or can it consist of several disjoint parts; are roads and paths going through a forest parts of the forest? Sorites vagueness on the other hand occurs when the threshold used to decide whether an object exhibits a certain property is illdefined: for example, how many trees are needed to make up a forest? Other prototypical examples of sorites vagueness are properties such as 'old', 'tall', 'warm', ... While we agree with [5] and [8] that supervaluation semantics may be a suitable formalism to handle conceptual vagueness, we adopt the view from [7] that sorites vagueness should be modelled by using fuzzy set theory. Since most geographic objects and relations are indeed susceptible to the sorites paradox (i.e. affected by sorites vagueness), we will typically represent vague regions by fuzzy sets and imprecise spatial relations by fuzzy relations.

A straightforward fuzzification of the definitions of the RCC- 8 is given in [12]. The starting point is an arbitrary symmetric fuzzy relation $C$ in $U$, satisfying a weak reflexivity property, namely $C(a, a)>0.5$ for every region $a$ from $U$. The fuzzy relation $P$ (part of) for example is defined 
by

$$
P(a, b)=\inf _{c \in U} I_{S_{M}}(C(c, a), C(c, b))
$$

where $a$ and $b$ are regions in $U$. However, many of the properties of the original RCC- 8 relations are lost in this approach. For example, as seen also in Figure 1, from $C(a, b)$ and $P(b, c)$ we conclude that $C(a, c)$ holds. When fuzzifying the RCC- 8 relations, it would be desirable that, for a particular t-norm $T, T(C(a, b), P(b, c)) \leq C(a, c)$ holds, which is a generalization of the aforementioned property. Using (20), no such property can be guaranteed, due to the poor choice of logical operators. Moreover, it is not clear how the definitions proposed in [12] should be applied to calculate the values of the fuzzy spatial relations between two given vague regions, e.g. represented as fuzzy sets, for particular definitions of $C$, as these definitions include infima and suprema ranging over $U$, which contains a potentially infinite amount of regions.

In [13], a generalization of the 9-intersection model based on $\alpha$-levels of fuzzy sets is suggested. However, the resulting membership degree of a given fuzzy spatial relation reflects the probability that this spatial relation holds, rather than a degree of compatibility. Moreover, the approach given in [13] is not suitable for spatial reasoning.

In [14], crisp spatial relations, based on the RCC, are defined between vague regions represented by fuzzy sets. This approach can only be used when the membership values of the fuzzy sets are taken from a finite universe. As for the egg-yolk calculus, this leads to a significant increase of the number of relations, since the exact number of relations is dependent on the cardinality of the finite set of membership values.

In [15], a generalization of the 9-intersection model is introduced using concepts from fuzzy topology; again an excessive amount (44) of crisp spatial relations between fuzzy sets are defined.

Apart from these generalizations of the RCC and 9intersection model, imprecise spatial relations between points have also been extensively studied. For example, $[16]$ is concerned with spatial relations such as " $x$ is located about 5 miles from $y$ in a northeasterly direction". Similarly, in [17] spatial relations such as " $x$ is close to $y$ " are considered. Analogously, imprecise spatial relations between (vague) regions have also been considered. For example, $[18]$ is concerned with expressing spatial relations between fuzzy regions, such as " $a$ is near $b$ " and " $a$ is located east of $b$ ". To this end, the direction and distance between two fuzzy sets are represented as fuzzy numbers instead of scalars.

However, to our knowledge, a general approach to imprecise qualitative spatial relations between regions, such as in the context of the RCC or the 9-intersection model, has not yet been considered.

\section{FuZzy Spatial RELATions}

\section{A. Definition}

Henceforth, let $T$ denote a left-continuous t-norm and $I_{T}$ its residual implicator. Let $C$ be a reflexive and symmetric fuzzy relation. Table II proposes our generalization of the spatial relations of the RCC; note that $\operatorname{NTP}(a, b)$ is introduced here merely as a shorthand for technical purposes (see below).

Most of these expressions are straightforward generalizations of the definitions in Table I. Note that the conjunction ' $\wedge$ ' is sometimes modelled by min (e.g. in $E Q(a, b)$ ) and sometimes by $T$ (e.g. in $O(a, b))$. This is because in the former case, the joint satisfaction of two independent constraints is evaluated, hence idempotency is required (recall that min is the only idempotent t-norm). The use of particular choices of $T$ other than min in the latter case guarantees, as we shall see, the preservation of several important properties.

To obtain the specific form of $\operatorname{TPP}(a, b)$ and $N T P P(a, b)$, first note that in the RCC, it holds that

$$
\begin{aligned}
& P(a, b) \wedge \neg(\exists c \in U)(E C(c, a) \wedge E C(c, b)) \\
\equiv & (\forall c \in U)(C(c, a) \Rightarrow O(c, b)) \\
\equiv & N T P(a, b)
\end{aligned}
$$

As a consequence, when $C$ is a crisp relation our definitions coincide with the original definitions of the RCC. Note that these definitions do not impose a restriction on how vague regions have to modelled, although we will typically use them when vague regions are modelled as fuzzy sets.

\section{B. Properties}

Below, we summarize some important properties concerning our fuzzy spatial relations with $S_{W}$ the Eukasiewicz t-conorm. These properties are valid when $C$ is an arbitrary reflexive and symmetric fuzzy relation in an arbitrary universe of regions $U$, and $a$ and $b$ are elements of $U$.

\section{Proposition 1:}

$$
\begin{aligned}
S_{W}(T P P(a, b), N T P P(a, b)) & \geq P P(a, b) \\
S_{W}(P P(a, b), E Q(a, b)) & \geq P(a, b) \\
S_{W}\left(P O(a, b), P(a, b), P P^{-1}(a, b)\right) & \geq O(a, b) \\
S_{W}(O(a, b), E C(a, b)) & \geq C(a, b) \\
S_{W}(E C(a, b), D C(a, b)) & \geq D R(a, b) \\
S_{W}(C(a, b), D R(a, b)) & =1
\end{aligned}
$$

Proposition 2:

$$
\begin{aligned}
P O(a, b) & \leq O(a, b) \\
T P P(a, b) & \leq P P(a, b) \\
N T P P(a, b) & \leq P P(a, b) \\
P P(a, b) & \leq P(a, b) \\
E Q(a, b) & \leq P(a, b) \\
P(a, b) & \leq O(a, b) \\
O(a, b) & \leq C(a, b) \\
E C(a, b) & \leq C(a, b) \\
E C(a, b) & \leq D R(a, b) \\
D C(a, b) & \leq D R(a, b)
\end{aligned}
$$


TABLE II

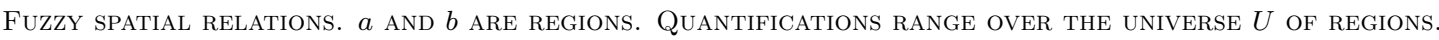

\begin{tabular}{lll}
\hline Name & Definition & \\
\hline disconnected from & $D C(a, b)$ & $=1-C(a, b)$ \\
part of & $P(a, b)$ & $=\inf _{c \in U} I_{T}(C(c, a), C(c, b))$ \\
proper part of & $P P(a, b)$ & $=\min (P(a, b), 1-P(b, a))$ \\
coincides with & $E Q(a, b)$ & $=\min (P(a, b), P(b, a))$ \\
overlaps with & $O(a, b)$ & $=\sup _{c \in U} T(P(c, a), P(c, b))$ \\
discrete from & $D R(a, b)$ & $=1-O(a, b)$ \\
partially overlaps with & $P O(a, b)$ & $=\min (O(a, b), 1-P(a, b), 1-P(b, a))$ \\
externally connects with & $E C(a, b)$ & $=\min (C(a, b), 1-O(a, b))$ \\
non-tangential part of & $N T P(a, b)$ & $=\inf \left(c \in U I_{T}(C(c, a), O(c, b))\right.$ \\
tangential proper part of & $T P P(a, b)$ & $=\min (P P(a, b), 1-N T P(a, b))$ \\
non-tangential proper part of & $N T P P(a, b)$ & $=\min (1-P(b, a), N T P(a, b))$ \\
\hline
\end{tabular}

These properties clearly extend the subsumption hierarchy in Figure 2. As we have mentioned, the relations of the RCC- 8 and of the RCC-5 are jointly exclusive and pairwise disjoint sets of relations. Again we can generalize this for our definitions.

Proposition 3: Let $R$ and $Q$ be two of the fuzzy relations $D C, E Q, E C, P O, T P P, N T P P, T P P^{-1}$ and $N T P P^{-1}$. It holds that

$$
T_{W}(R(a, b), Q(a, b))=0
$$

Proposition 4:

$$
\begin{gathered}
S_{W}(D C(a, b), E Q(a, b), E C(a, b), P O(a, b), \\
\operatorname{TPP}(a, b), \operatorname{NTPP}(a, b), \operatorname{TPP}^{-1}(a, b), \\
\left.N T P P^{-1}(a, b)\right)=1
\end{gathered}
$$

Proposition 5: Let $R$ and $Q$ be two of the fuzzy relations $D R, E Q, P O, P P$ and $P P^{-1}$. It holds that

$$
T_{W}(R(a, b), Q(a, b))=0
$$

Proposition 6:

$$
\begin{gathered}
S_{W}(D R(a, b), E Q(a, b), P O(a, b), \\
\left.P P(a, b), P P^{-1}(a, b)\right)=1
\end{gathered}
$$

Proposition 7: The fuzzy relations $P, O$ and $E Q$ are reflexive, while the fuzzy relations $D C, P P, D R, P O, E C$, $T P P$ and NTPP are irreflexive.

\section{Spatial Reasoning}

To facilitate spatial reasoning (that is, the inference of new information from given spatial relations) with the RCC-8 relations, in [19] a transitivity table (or composition table) has been introduced. The purpose of such a table is to specify, for each pair $R, Q$ of spatial relations from the RCC-8, the disjunction of all relations $A$ such that $A(a, c)$ holds if $R(a, b)$ and $Q(b, c)$ for arbitrary regions $a, b$ and $c$.

For example, from $D C(a, b) \wedge E C(b, c)$ follows that $D R(a, c) \vee P O(a, c) \vee P P(a, c)$. It holds that

$$
D R(a, c) \vee P O(a, c) \vee P P(a, c) \equiv \neg P^{-1}(a, c)
$$

Similarly, all disjunctions in the transitivity table given in [19] are equivalent to conjunctions of $C, P, P^{-1}, O, N T P$, $N T P^{-1}, \neg C, \neg P, \neg P^{-1}, \neg O, \neg N T P$ and $\neg N T P^{-1}$. Using our definitions, when $C$ is a fuzzy relation (generalizations of) these equivalences do not longer hold. However, we still have

$$
1-P^{-1}(a, c) \leq S_{W}(D R(a, c), P O(a, c), P P(a, c))
$$

Hence $1-P^{-1}(a, c)$ is a stronger conclusion (that is, a lower bound for the disjunction). Table III is a generalized transitivity table for our definitions, which is because of this observation formulated in terms of $C, 1-C, P, 1-P$, ... instead of disjunctions. For example, the entry on the second row, first column should be interpreted as

$$
T_{W}(1-C(a, b), C(b, c)) \leq 1-P^{-1}(a, c)
$$

Furthermore, our generalized definitions of the RCC- 8 relations are formulated in terms of the minimum of some of the fuzzy relations from Table III. To support spatial reasoning with $\mathrm{RCC}-8$ relations, we can make use of the following lemma.

Lemma 1: For an arbitrary t-norm $T$ and $x, y$ and $z$ in $[0,1]$, it holds that

$$
T(\min (x, y), z) \leq \min (T(x, z), T(y, z))
$$

For example, using this lemma we obtain, for regions $a, b$ 
TABLE III

Transitivity table

\begin{tabular}{|c|cccccc|}
\hline & $C$ & $1-C$ & $P$ & $P^{-1}$ & $1-P$ & $1-P^{-1}$ \\
\hline$C$ & 1 & $1-P$ & $C$ & 1 & 1 & 1 \\
$1-C$ & $1-P^{-1}$ & 1 & $1-P^{-1}$ & $1-C$ & 1 & 1 \\
$P$ & 1 & $1-C$ & $P$ & 1 & 1 & $1-P^{-1}$ \\
$P^{-1}$ & $C$ & $1-P$ & $O$ & $P^{-1}$ & $1-P$ & 1 \\
$1-P$ & 1 & 1 & 1 & $1-P$ & 1 & 1 \\
$1-P^{-1}$ & 1 & 1 & $1-P^{-1}$ & 1 & 1 & 1 \\
$O$ & 1 & $1-P$ & $O$ & 1 & 1 & 1 \\
$1-O$ & $1-N T P^{-1}$ & 1 & $1-P^{-1}$ & $1-O$ & 1 & 1 \\
$N T P$ & 1 & $1-C$ & $N T P$ & 1 & 1 & $1-P^{-1}$ \\
$N T P^{-1}$ & $O$ & $1-P$ & $O$ & $N T P^{-1}$ & $1-P$ & 1 \\
$1-N T P$ & 1 & 1 & 1 & $1-N T P$ & 1 & 1 \\
$1-N T P^{-1}$ & 1 & 1 & $1-N T P^{-1}$ & 1 & 1 & 1 \\
\hline
\end{tabular}

\begin{tabular}{|c|cccccc|}
\hline & $O$ & $1-O$ & $N T P$ & $N T P^{-1}$ & $1-N T P$ & $1-N T P^{-1}$ \\
\hline$C$ & 1 & $1-N T P$ & $O$ & 1 & 1 & 1 \\
$1-C$ & $1-P^{-1}$ & 1 & $1-P^{-1}$ & $1-C$ & 1 & 1 \\
$P$ & 1 & $1-O$ & $N T P$ & 1 & 1 & $1-N T P^{-1}$ \\
$P^{-1}$ & $O$ & $1-P$ & $O$ & $N T P^{-1}$ & $1-N T P$ & 1 \\
$1-P$ & 1 & 1 & 1 & $1-P$ & 1 & 1 \\
$1-P^{-1}$ & 1 & 1 & $1-P^{-1}$ & 1 & 1 & 1 \\
$O$ & 1 & $1-P$ & $O$ & 1 & 1 & 1 \\
$1-O$ & $1-P^{-1}$ & 1 & $1-P^{-1}$ & $1-C$ & 1 & 1 \\
$N T P$ & 1 & $1-C$ & $N T P$ & 1 & 1 & $1-P^{-1}$ \\
$N T P^{-1}$ & $O$ & $1-P$ & $O$ & $N T P^{-1}$ & $1-P$ & 1 \\
$1-N T P$ & 1 & 1 & 1 & $1-P$ & 1 & 1 \\
$1-N T P^{-1}$ & 1 & 1 & $1-P^{-1}$ & 1 & 1 & 1 \\
\hline
\end{tabular}

and $c$ in $U$,

$$
\begin{aligned}
& T_{W}(D C(a, b), E C(b, c)) \\
= & T_{W}(1-C(a, b), \min (C(b, c), 1-O(b, c))) \\
\leq \quad & \min \left(T_{W}(1-C(a, b), C(b, c)),\right. \\
& \left.T_{W}(1-C(a, b), 1-O(b, c))\right)
\end{aligned}
$$

From Table III we have

$$
\begin{aligned}
& \leq \min \left(1-P^{-1}(a, c), 1\right) \\
& =1-P^{-1}(a, c) \\
& =1-P(c, a)
\end{aligned}
$$

Hence from $a$ disconnected from $b$ and $b$ externally connected with $c$, we concluded that $c$ is not a part of $a$. In general, we can apply the following algorithm:

1. Assume two fuzzy spatial relations $R$ and $Q$ are given that can be written as

$$
\begin{aligned}
& R=\min \left(r_{1}, \ldots, r_{n}\right) \\
& Q=\min \left(q_{1}, \ldots, q_{m}\right)
\end{aligned}
$$

where $r_{i}$ and $q_{j}(1 \leq i \leq n, 1 \leq j \leq m)$ are $C, 1-C, P$, $P^{-1}, 1-P, 1-P^{-1}, O, 1-O, N T P, N T P^{-1}, 1-N T P$ or $1-N T P^{-1}$. This applies, among others, to all RCC -8 and $\mathrm{RCC}-5$ relations.

2. Repeatedly applying Lemma 1 yields

$$
\begin{aligned}
& T_{W}(R(a, b), Q(b, c)) \\
= & T_{W}\left(\min _{i=1}^{n} r_{i}(a, b), \min _{j=1} q_{j}(b, c)\right) \\
\leq & \min _{i=1}^{n} \min _{j=1}^{m} T_{W}\left(r_{i}(a, b), q_{j}(b, c)\right)
\end{aligned}
$$

3. For each $i$ and each $j$, use Table III to obtain a conclu- 
sion of the form

$$
T_{W}\left(r_{i}(a, b), q_{j}(b, c)\right) \leq t_{i j}(a, c)
$$

Hence we obtain

$$
T_{W}(R(a, b), Q(b, c)) \leq \min _{i=1}^{n} \min _{j=1}^{m} t_{i j}(a, c)
$$

4. Use Proposition 2 to obtain a minimal subset $A$ of $\left\{t_{i j}(a, c) \mid 1 \leq i \leq n, 1 \leq j \leq m\right\}$ for which it holds that

$$
\min _{i=1}^{n} \min _{j=1}^{m} t_{i j}(a, c)=\min _{t \in A} t(a, c)
$$

5. We conclude

$$
T_{W}(R(a, b), Q(b, c)) \leq \min _{t \in A} t(a, c)
$$

It can be shown that if $C$ is a crisp relation, the deductions made for the RCC-8 relations using this algorithm are equivalent to the deductions made using the transitivity table introduced in [19], hence our spatial reasoning algorithm is a sound generalization of spatial reasoning within the RCC-8.

\section{Fuzzy Spatial Relations Between FuzZy REGIONS}

In this section, we provide a concrete definition for the fuzzy spatial relations from Table II for a particular choice of $C$ and a particular representation of a vague region.

Since we want to model imprecise spatial relations, we need a way to model the concept of nearness. Due to the fuzzy nature of this concept, we will represent nearness as a fuzzy relation, by expressing that two objects are near to each other to the degree that their locations are approximately equal. We first consider approximate equality of point locations; afterwards we will show how such a measure for approximate equality of point locations can be used to define imprecise spatial relations between regions.

Recall that a fuzzy $T$-equivalence relation in $X$ is a reflexive, symmetric fuzzy relation $R$ in $X$ that satisfies $T$ transitivity, that is

$$
T(R(x, y), R(y, z)) \leq R(x, z)
$$

for all $x, y$ and $z$ in $X$. Clearly, if $R$ is a crisp relation, then $R$ is an equivalence relation in $X$. Hence fuzzy $T$-equivalence relations seem to be an ideal candidate to model approximate equality, and thus nearness. However, as pointed out in [20], in some contexts we want to express that, for $x_{i}$ in $X, i=\{1, \ldots, n\}, x_{1}$ and $x_{2}$ are approximately equal to degree $1, x_{2}$ and $x_{3}$ are approximately equal to degree $1, \ldots, x_{n-1}$ and $x_{n}$ are approximately equal to degree 1 , but $x_{1}$ and $x_{n}$ are not approximately equal at all. Using fuzzy $T$-equivalence relations this is impossible, because of their $T$-transitivity. Therefore, to model approximate equality, the notion of a resemblance relation has been introduced in [20] and [21]. Recall that $d$ is a pseudometric on $X$ if $d(x, x)=0, d(x, y)=d(y, x)$ and $d(x, y)+d(y, z) \geq d(x, z)$ for all $x, y$ and $z$ in $X$. A fuzzy relation $E$ in $X$ is called a resemblance relation w.r.t. a pseudometric $d$ on $X$ if for all $x, y, z$ and $u$ in $X$

$$
\begin{gathered}
E(x, x)=1 \\
d(x, y) \leq d(z, u) \Rightarrow E(x, y) \geq E(z, u)
\end{gathered}
$$

As a consequence of (52), any resemblance relation is also symmetric. For example, let $\mathcal{P}=\mathbb{R}^{2}$ and let $d$ be the Euclidean metric. It can be shown that the fuzzy relation $E$ in $\mathbb{R}$ defined for all $a$ and $b$ in $\mathbb{R}$ as

$$
E(a, b)= \begin{cases}1 & \text { if } d(a, b)<5 \\ 0 & \text { if } d(a, b)>10 \\ \frac{10-d(a, b)}{5} & \text { otherwise }\end{cases}
$$

is a resemblance relation w.r.t. $d$.

A straightforward generalization of the intended meaning of $C$ in the RCC is to express, for $A$ and $B$ fuzzy sets in the universe $\mathcal{P}$ of points, the degree to which there exists a point in $A$ which is approximately equal to a point in $B$, i.e.

$$
C(A, B)=A \circ_{T_{W}} E \circ_{T_{W}} B
$$

Now if we represent a region as a normalised fuzzy set in $\mathcal{P}$, we have the following important result.

Proposition 8: If $U$ is the set of all normalised fuzzy sets in a universe $\mathcal{P}$, and $C$ is defined by (54) for all fuzzy sets $A$ and $B$ in $\mathcal{P}$, then it holds that

$$
\begin{aligned}
P(A, B) & =\operatorname{incl}\left(E \downarrow_{I_{W}} \uparrow_{T_{W}} A, E \downarrow_{I_{W}} \uparrow_{T_{W}} B\right) \\
O(A, B) & =\operatorname{overl}\left(E \downarrow_{I_{W}} \uparrow_{T_{W}} A, E \downarrow_{I_{W}} \uparrow_{T_{W}} B\right) \\
\operatorname{NTP}(A, B) & =\operatorname{incl}\left(E \uparrow_{T_{W}} A, E \downarrow_{I_{W}} \uparrow_{T_{W}} B\right)
\end{aligned}
$$

In particular, when $A$ and $B$ are $E$-closed normalised fuzzy sets in $\mathcal{P}$, we obtain

$$
\begin{aligned}
P(A, B) & =\operatorname{incl}(A, B) \\
O(A, B) & =\operatorname{overl}(A, B) \\
\operatorname{NTP}(A, B) & =\operatorname{incl}\left(E \uparrow_{T_{W}} A, B\right)
\end{aligned}
$$

In other words, if $A$ and $B$ are $E$-closed, the fuzzy relations $P$ and $O$ correspond to the classical definitions of inclusion and overlap of fuzzy sets.

Proposition 9: Under the same assumptions as in Proposition 8 , if additionally $E$ is $T_{W}$-transitive (i.e. $E$ is a $T_{W^{-}}$ equivalence relation), it holds that

$$
C(A, B)=O(A, B)
$$

for all fuzzy sets $A$ and $B$ in $U$.

As a consequence, also $\operatorname{NTPP}(A, B)=\operatorname{PP}(A, B)$. Hence, when we use a $T_{W}$-equivalence relation, we can only distinguish between the five relations of the RCC-5! 


\section{Future Work And Conclusion}

We intend to apply the ideas presented in this paper to build a focused geographical information retrieval system in which users may express their information need in natural language. Like in question answering systems, and in contrast with traditional search engines, the system should return exactly the information that was requested, instead of a list of possibly relevant documents. The reasoning part of such a geographical IR system boils down to determining the qualitative relation that holds between two entities. For example to answer the query "give me addresses and opening hours of bookshops in London's West End", we need to ascertain whether or not $P P$ holds between a particular bookshop and London's West End.

Most of the relevant information on the Web is of a qualitative nature. We may find documents stating that the West End is located in the centre of London, that it is adjacent to the City of London, or that it contains Leicester Square, Covent Garden, Oxford Street, etc. For example, consider the following snippets:

1. Waterstone's (Located on Piccadilly) 203-206 Piccadilly, W1J, (Books) Close by. The biggest bookshop in Europe, Waterstone's huge floorspace in an ... ${ }^{1}$

2. And praise is also due to those far-sighted anarchists who strolled up Piccadilly and other West End avenues, taking thousands of demonstrators away from ... ${ }^{2}$

This can be represented as $P P$ (Waterstone's,Piccadilly) and $P P$ (Piccadilly,West_End), from which we can deduce, using the RCC, that PP(Waterstone's, West_End) holds.

Another possibility to determine if a bookshop is located in the West End, is to use geocoding techniques to map the address of the bookshop to latitude-longitude coordinates. This requires a suitable representation of the extent of the West End. For some regions or districts, such a representation can be obtained using a technique described in [22]. Since regions or districts such as the West End tend to be vague, their extent can be represented by a fuzzy set. The characterizations in Proposition 8 can then be used to calculate the degree to which a particular spatial relation holds between two regions, or between a point and a region.

In practice, it is likely that we will need to combine both techniques, i.e. extracting qualitative relations and geocoding, because for many regions or districts we may find too little information to construct an accurate representation. Whenever this is the case we need to draw inferences based on vague qualitative information such as " $P P$ (Paddington, West End) holds at least to degree 0.6", which can be done using the inequalities of Table III.

In conclusion, the fuzzy spatial relations introduced in this paper provide a sound, and workable, generalization of the RCC that lends itself ideally to representing, and reasoning with, imprecise qualitative relations between vague regions.

\footnotetext{
${ }^{1}$ http://www . londontown. com/LondonStreets/piccadilly_1e1. html, accessed December 4, 2005

${ }^{2}$ http://www. londonclasswar.org/london_calling_2003.htm, accessed December 4, 2005
}

\section{REFERENCES}

[1] D.A. Randell, Z. Cui and A.G. Cohn, A spatial logic based on regions and connection, Proc. of the 3rd Int. Conf. on Knowledge Representation and Reasoning, 165-176, 1992.

[2] M.J. Egenhofer and R. Franzosa, Point-set topological spatial relations, Int. J. Geographical Information Systems, 5:161-174, 1991.

[3] L.A. Zadeh, Fuzzy sets, Information and Control, 8:338-353, 1965.

[4] S. Schockaert, M. De Cock and C. Cornelis, Relatedness of fuzzy sets, Int. J. Intelligent and Fuzzy Systems, 16(4), 2005.

[5] B. Bennett, What is a forest? On the vagueness of certain geographic concepts, Topoi, 20:189-201, 2001.

[6] T. Bittner and J.G. Stell, Vagueness and rough location, Geoinformatica, 6:99-121, 2002.

[7] P. Fisher, Sorites paradox and vague geographies, Fuzzy Sets and Systems, 113:7-18, 2000.

[8] A.C. Varzi, Vagueness in geography, Philosophy \& Geography, 4:49-65, 2001.

[9] A.G. Cohn and N.M. Gotts, The 'egg-yolk' representation of regions with indeterminate boundaries, Geographic Objects with Indeterminate Boundaries (P.A. Burrough and A.U. Frank, eds.), Taylor and Francis Ltd., 171-187, 1996.

[10] E. Clementini and P. Di Felice, Approximate topological relations," Int. J. Approximate Reasoning, 16:173-204, 1997.

[11] A.J. Roy and J.G. Stell, Spatial relations between indeterminate regions, Int. J. Approximate Reasoning, 27:205-234, 2001.

[12] A. Esterline, G. Dozier and A. Homaifar, Fuzzy spatial reasoning, Proc. of the 1997 Int. Fuzzy Systems Association Conf., 162-167, 1997.

[13] F.B. Zhan, Approximate analysis of binary topological relations between geographic regions with indeterminate boundaries, Soft Computing, 2:28-34, 1998.

[14] Y. Li and S. Li, A fuzzy sets theoretic approach to approximate spatial reasoning, IEEE Trans. on Fuzzy Systems, 12:745-754, 2004.

[15] X. Tang and W. Kainz, Analysis of topological relations between fuzzy regions in a general fuzzy topological space, Proc. of the Symposium on Geospatial Theory, Processing and Applications, 2002, available at http://www.isprs.org/commission4/ proceedings

[16] S. Dutta, Approximate spatial reasoning: integrating qualitative and quantitative constraints, Int. J. Approximate Reasoning, 5:307-331, 1991.

[17] H.W. Guesgen and J. Albrecht, Imprecise reasoning in geographic information systems, Fuzzy Sets and Systems, 113:121$131,2000$.

[18] D. Altman, Fuzzy set theoretic approaches for handling imprecision in spatial analysis, Int. J. Geographical Information Systems, 8:271-289, 1994.

[19] Z. Cui, A.G. Cohn and D.A. Randell, Qualitative and topological relationships, Proc. of the Third International Symposium on Advances in Spatial Databases, Lecture Notes in Computer Science, 692:296-315, 1993.

[20] M. De Cock and E.E. Kerre, On (un) suitable fuzzy relations to model approximate equality, Fuzzy Sets and Systems, 133:137153, 2003.

[21] M. De Cock and E.E. Kerre Why fuzzy $\mathcal{T}$-equivalence relations do not resolve the Poincaré paradox, and related issues, Fuzzy Sets and Systems, 133:181-192, 2003.

[22] S. Schockaert, M. De Cock and E.E. Kerre, Automatic Acquisition of Fuzzy Footprints, Lecture Notes in Computer Science, 3762:1077-1086, 2005. 